\title{
Recruitment of Participants and Delivery of Online Mental Health Resources for Depressed Individuals Using Tumblr: Pilot Randomized Control Trial
}

Erin Kelleher ${ }^{1}$, BS; Megan Moreno ${ }^{1,2}$, MD, MPH, MSEd; Megan Pumper Wilt ${ }^{1}$, BA

${ }^{1}$ Seattle Children's Research Institute, Center for Child Health, Behavior and Development, Seattle, WA, United States

${ }^{2}$ University of Washington, Seattle, WA, United States

Corresponding Author:

Erin Kelleher, BS

Seattle Children's Research Institute

Center for Child Health, Behavior and Development

2001 8th Ave

Seattle, WA, 98121

United States

Phone: 18479329259

Email: erin.kelleher6@gmail.com

\section{Abstract}

Background: Adolescents and young adults frequently post depression symptom references on social media; previous studies show positive associations between depression posts and self-reported depression symptoms. Depression is common among young people and this population often experiences many barriers to mental health care. Thus, social media may be a new resource to identify, recruit, and intervene with young people at risk for depression.

Objective: The purpose of this pilot study was to test a social media intervention on Tumblr. We used social media to identify and recruit participants and to deliver the intervention of online depression resources.

Methods: This randomized pilot intervention identified Tumblr users age 15-23 who posted about depression using the search term “\#depress". Eligible participants were recruited via Tumblr messages; consented participants completed depression surveys and were then randomized to an intervention of online mental health resources delivered via a Tumblr message, while control participants did not receive resources. Postintervention online surveys assessed resource access and usefulness and control groups were asked whether they would have liked to receive resources. Analyses included $t$ tests.

Results: A total of 25 participants met eligibility criteria. The mean age of the participants was 17.5 (SD 1.9) and 65\% were female with average score on the Patient Health Questionnaire-9 of 17.5 (SD 5.9). Among the 11 intervention participants, 36\% (4/11) reported accessing intervention resources and 64\% (7/11) felt the intervention was acceptable. Among the 14 control participants, only $29 \%$ (4/14) of reported that receiving resources online would be acceptable $(P=.02)$. Participants suggested anonymity and ease of use as important characteristics in an online depression resource.

Conclusions: The intervention was appropriately targeted to young people at risk for depression, and recruitment via Tumblr was feasible. Most participants in the intervention group felt the social media approach was acceptable, and about a third utilized the online resources. Participants who had not experienced the intervention were less likely to find it acceptable. Future studies should explore this approach in larger samples. Social media may be an appropriate platform for online depression interventions for young people.

(JMIR Res Protoc 2018;7(4):e95) doi: 10.2196/resprot.9421

\section{KEYWORDS}

adolescents; depression; intervention; social media; tumblr 


\section{Introduction}

Depression is among the most common illnesses affecting adolescents and young adults but many do not seek clinical care or know about available resources [1-3]. Previous studies have illustrated several barriers to mental health care among young people, including recognition of a problem, access to care, fear of stigma if care is accessed, and identification of young people-specific resources [4-6]. Many of the services offered do not address the needs of young people during the later years of adolescence and young adulthood [4]. Given these barriers to mental health care, innovative solutions are needed. Online approaches may be one such solution.

Due to the continuous rise in social media use among young people, researchers are investigating the impact and influence of online mental health resources $[7,8]$. The vast majority of young people are online and most of these young people use social media to express themselves and get social support [9-11]. Many social media users report feeling more comfortable self-disclosing information online rather than in person [11]. Previous studies suggest depression symptom disclosures on social media are common and are associated with offline depression symptoms [12,13]. One study found one-third of public college Facebook profiles displayed at least one reference to a depression symptom and $2.5 \%$ of Facebook profiles fit the Diagnostic and Statistical Manual (DSM-IV) criteria for a major depressive episode [13]. Thus, it is possible that social media posts could be used to identify young people at risk for depression based on posting references to depression symptoms. In this way, young people could be identified and even provided resources or support online.

There are several ways in which online and social media interventions present opportunities to reach and engage with young people $[9,14,15]$. Online and social media interventions often cost less and allow researchers to reach a more diverse population [14,15] . Reaching young people is a critical challenge as young people are the least likely age group to have a primary care provider and a majority do not seek professional help for mental health problems $[2,6,16]$. Previous online intervention studies had more participation from underrepresented and underserved individuals compared to traditional intervention methods [14,17]. Online interventions allow individuals to access the intervention anytime without geographical and time barriers. A majority of the successful online intervention studies used structured online programs that included education and coping strategies as their means of intervention [3,8,18-21]. Many young people prefer online intervention options because they offer privacy, anonymity, 24-hour accessibility, and avoid face-to-face interactions $[9,22]$.

While previous online interventions have addressed alcohol use, sexual behaviors, depression, and other mental health disorders, none of those interventions offered online resources for adolescents to use independently and a majority of studies did not use social media for participant identification and recruitment $[3,21,23]$. The purpose of this study was to determine whether a social media intervention offering resources to young people displaying references to depression appropriately targeted young people with depression and was accessed by, and deemed acceptable by, young people.

\section{Methods}

\section{Study Setting}

This randomized control pilot intervention occurred from October 2014 to December 2015 on Tumblr [24]. Tumblr is a popular social media site among young people that allows profiles to be created and displayed anonymously [7]. An email address and username is the only information Tumblr requires to create an account. Tumblr users are not required to display identifying information on their profile page. The email address provided to create a profile is not visible to other users. Thus, users can post personal multimedia content labeled with hashtags with fewer restrictions compared to Facebook or Twitter. Similar to Facebook and Twitter, users can repost, like, and comment on other user's posts. A majority of Tumblr blogs are publically available and profiles and posts can be viewed without having a Tumblr profile [25]. The Western Institutional Review Board approved this pilot study.

\section{Participants}

The goal for this pilot study was to identify participants who displayed depression symptoms on social media. The target sample size was 45 for this pilot test. Potential participants were identified via a post on Tumblr using the search term \#depress to encompass both key words "depression" and "depressed," and search filter of "most recent posts."

A codebook from a previous study was used to determine whether the post displayed a reference to depression [12]. The codebook was created using the DSM-IV diagnostic criteria for a major depressive episode (see Table 1). Examples of posts eligible for participation included: "I feel like I am not good enough for anyone \#depress \#sad;" "I can't do anything right. Straight A's still isn't good enough for my parents. I'm so exhausted and I am hurting physically and emotionally. \#depress \#zzz \#alone \#depression"; and a picture of cuts on an arm with the caption, "they thought I was fine. \#alone \#depress \#tears \#die".

After a post was determined to meet the depression codebook criteria, the profile of the individual that created the post was reviewed. Participants whose Tumblr profiles were in English, displayed their profile age as between 15 and 23 years, and displayed one or more depression symptoms consistent with the DSM-IV in their Tumblr post within the last 14 days were considered eligible $[13,26]$.

Individuals with Tumblr profiles who displayed other mental health comorbidities (ie, \#bipolar) were excluded. Additional inclusion criteria were the ability to receive private Tumblr messages and presence of timestamps on profile posts. 
Table 1. Depression codebook criteria.

\begin{tabular}{|c|c|c|}
\hline Category & Terms or phrases to include & Terms or phrases to exclude \\
\hline Depressed mood & Sad, empty, crying, tearful, alone, lonely, sad face emoticon & "I had a bad day", "FML" \\
\hline Decreased interest or pleasure in activities & Not having fun, don't feel like doing anything, giving up & \\
\hline Increase/decrease in appetite & $\begin{array}{l}\text { No appetite, don't feel like eating, can't stop eating, eating } \\
\text { everything in sight }\end{array}$ & $\begin{array}{l}\text { "I ate too much at McDonalds this week- } \\
\text { end," references to poor eating habits rather } \\
\text { than changes in appetite }\end{array}$ \\
\hline Sleep problems & $\begin{array}{l}\text { Sleeping too much, slept more than } 10 \text { hours, fatigue, tired, } \\
\text { exhausted }\end{array}$ & \\
\hline Psychomotor agitation/retardation & Feeling slow & \\
\hline Loss of energy & Can't get anything done, no motivation & \\
\hline Indecisiveness & $\begin{array}{l}\text { Can't decide on something, don't feel like deciding, can't } \\
\text { make up mind }\end{array}$ & \\
\hline $\begin{array}{l}\text { Recurrent thoughts of death or suicidal } \\
\text { ideation }\end{array}$ & Thinking of ways to commit suicide, references to jumping & \\
\hline Difficulty concentrating & Can't study, can't finish work, can't concentrate & $\begin{array}{l}\text { Don't want to concentrate, can't concentrate } \\
\text { because of activity (TV, friends, Facebook) }\end{array}$ \\
\hline
\end{tabular}

\section{Intervention}

The intervention tested in this research protocol was designed to be delivered online and provide online depression resources. A resource sheet was developed in consultation with an adolescent health mental health expert listing mental health resources that were nationally available, free, online, and publicly accessible (Multimedia Appendix 1). Not all of the resources provided were explicitly for young people, however some of the resources had specific pages or topics specifically for young people. Because a majority of young people do not seek help or know how to access mental health resources, the resource sheet offered a variety of depression resources for participants to access [1]. The resources included chat rooms, information-based websites, hotlines, and means to find a mental health professional for depression. A majority of the resources could be accessed 24 hours a day. The resource sheet was delivered using a study team Tumblr profile via a private Tumblr message explaining the study and the research team (Multimedia Appendix 1). Participants were asked to message the research group via Tumblr if they did not wish to be contacted again by the research group.

\section{Procedures}

After eligible individuals' Tumblr profiles were identified and randomized, the username, profile URL, post with the depression symptom reference, and hashtags of participants were stored in an excel spreadsheet. Participants were randomized using a randomization website [27]. The numbers 1 through 45 were randomized into intervention and control numbers. Participants were assigned numbers in the order their post appeared on Tumblr. Intervention participants received a resource list as the intervention while the participants in the control group were not initially contacted. One month after the intervention was delivered, a private Tumblr message was sent to both groups that included a link to the secure, anonymous online survey and consent form. Participants were provided information about the research group and how to contact the group, anonymity of the survey, how long the data would be stored, and how to exit the survey. There was one question per page with a maximum of 22 questions depending on how the participant answered specific questions. Participants were not required to provide an answer for any of the questions and were provided a back button to change previous answers. Due to the anonymity of the survey, researchers were not able to identify which participants completed the survey. IP addresses were verified to prevent duplicate entries from the same user. Intervention procedures are described in Figure 1. Participants who completed the survey were provided a $\$ 10$ gift card.

\section{Variables}

\section{Feasibility of Recruitment}

In order to assess the feasibility of recruiting teens at risk for depression, our survey included the Patient Health Questionnaire-9 (PHQ-9) [28]. The PHQ-9 is a 9-item measure that assesses DSM-IV criteria for depression within the last two weeks and is commonly used as a clinical screening tool across the United States with the following Likert scaled responses $(0=$ Not at all, $1=$ Several days, $2=$ More than half days, and $3=$ Nearly every day). The scores on the PHQ- 9 were computed and categories of depression level were established based on the PHQ-9 scoring guidelines: none to minimal depression (0-4 score), mild depression (5-9 score), moderate depression (10-14 score), moderate to severe depression (15-19 score), and severe depression (20-27 score). We hypothesized that if the average PHQ-9 score was in the range of mild to moderate depression, our search strategy was a feasible method of identifying teens at-risk for depression. 
Figure 1. Flowchart of study design. $P$ values calculated using an unpaired $t$ test. Bolded values indicate significant values. PHQ-9: Patient Health Questionnaire-9.

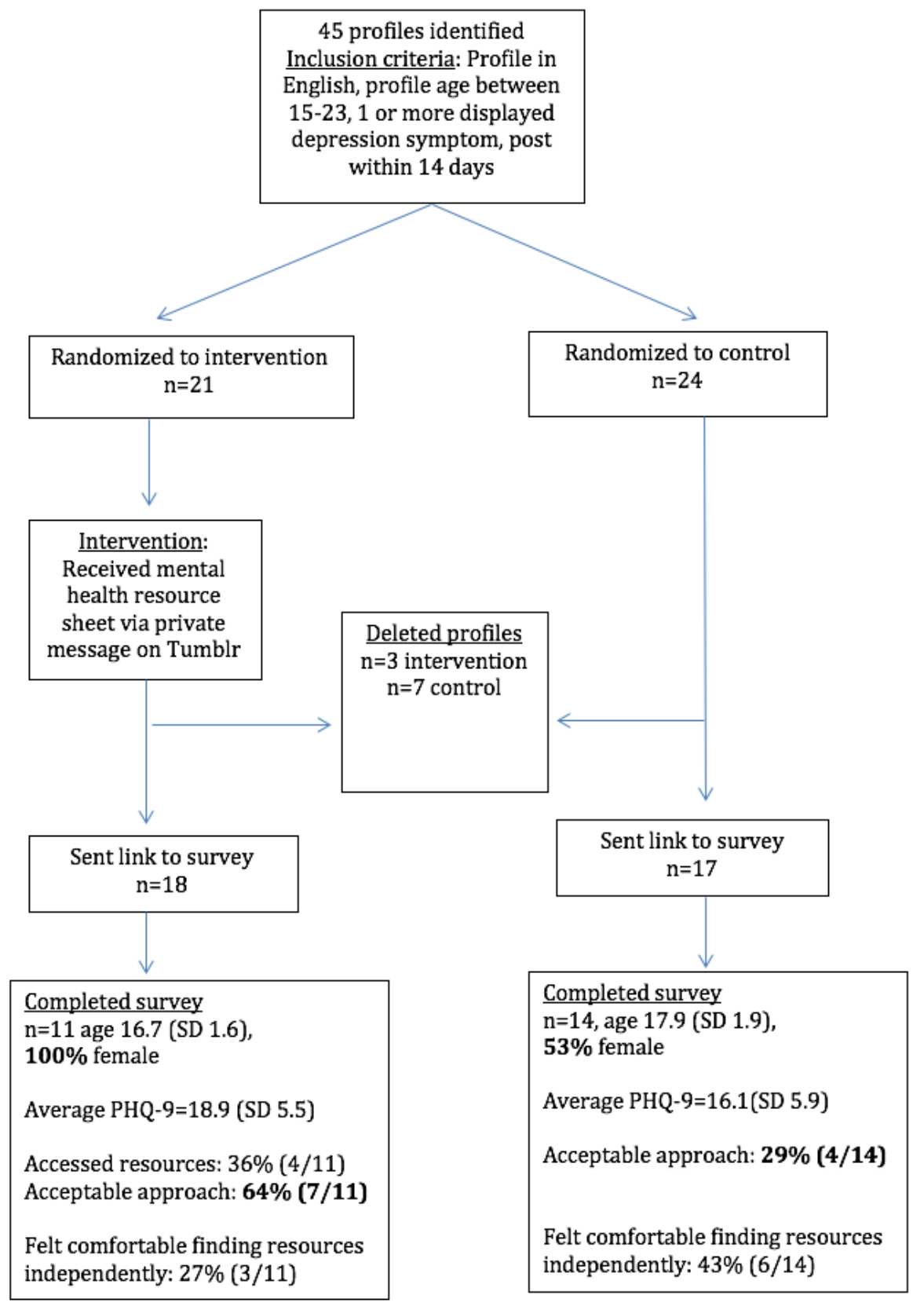

\section{Access to intervention resources}

To assess whether intervention participants accessed resources, we asked the intervention group on the follow-up survey to describe resources the participant accessed or viewed and which ones they found helpful. To understand participants' access to online mental health resources in general, all participants were asked whether they knew of, felt comfortable accessing, or used other online depression resources in the past.

\section{Acceptability of intervention approach}

For both groups, the survey assessed acceptability of the approach of sending resources via Tumblr. The control group survey asked if they would have liked to receive depression resources and if the intervention approach would be acceptable.

\section{Participant suggestions}

All participants were asked to provide suggestions on key components of online mental health interventions that would support their acceptability.

\section{Analysis}

Descriptive data were summarized; group comparisons and comparison of PHQ-9 scores postintervention were both conducted using an unpaired $t$ test. There was no blinding for this study.

\section{Results}

\section{Participants}

Among the 45 participants initially selected, 21 were randomized to the intervention. A total of 10 ( 3 intervention) participants 
deleted their profiles before receiving a survey. There were 25 completed surveys, $65 \%(16 / 25)$ were female and were an average age of 17.5 years (SD 1.9; Figure 1). The average PHQ-9 score was 17.5 (SD 5.4), not differing among groups. None of the participants messaged the researchers to not be contacted again.

\section{Intervention Group}

Among intervention participants $(n=11), 64 \%(7 / 11)$ of the participants found this intervention method to be an acceptable approach. Roughly one-third (4/11) of participants accessed intervention resources. Of those 4 participants, $100 \%$ (4) felt the approach was acceptable and 75\% (3/4) answered they would likely use the resources again. The Depression Chat Rooms, IM ALIVE, and National Suicide Hotline were the resources accessed. Only 27\% (3/11) felt comfortable finding resources independently. Before receiving the resources, half of the participants that accessed the resources did not know of other online resources for mental health (2/4).

\section{Control Group}

Among control group participants $(n=14)$, only $14 \%(2 / 14)$ of the control group would have wanted to receive resources online and 29\% (4/14) of control group participants reported they thought the intervention would be an acceptable approach. There was a statistically significant difference between the acceptability of the intervention approach between the intervention and control groups $(P=.02)$. Although $71 \%(10 / 14)$ of control participants had used online resources for mental health, only $43 \%(6 / 14)$ felt comfortable finding resources independently. Common resources accessed by control participants included WedMD, psychcentral, anonymous chat groups and hotlines, and Tumblr.

\section{Participant Suggestions}

Overall, participants in both groups felt anonymity, supportiveness, and ease of use as important characteristics in an online depression resource. Participants in the control group found websites with young people going through similar experiences and chat groups to be the most helpful resources. A majority (9/11) of participants in the intervention group were not sure or did not have resources to suggest. The two suggestions were to offer cognitive behavior therapy resources and www.emotionalbaggage.com.

\section{Discussion}

\section{Principal Findings}

The research protocol tested in this pilot intervention study included an online mental health resource sheet and used Tumblr to identify teens that posted about depression as well as to deliver the resource. We found that the intervention was appropriately targeted to young people at risk for depression and recruitment via Tumblr was feasible.

The young people in this study had an average PHQ-9 score of 17.5 , which can be interpreted as moderately severe depression [28]. Thus, identifying participants based on depression posts on Tumblr targeted the appropriate population for this study. Findings are similar to previous work in which depression displays on Facebook were associated with self-reported depression symptoms [13]. Therefore, displayed depression references on Tumblr could be a helpful tool in identifying young people at risk for depression.

Over one-third of participants in the intervention group accessed the online resources provided. The participants that used the resources found the resources to be helpful and stated they would use them in the future. Participants utilized the anonymous chat rooms and hotlines, which were similar to many of the resources accessed independently by the control group. However, it is important to highlight that Tumblr and WebMD, though unverified by a mental health expert, were considered depression resources for some participants in the control group. It is possible that participants in the control group did not find this approach acceptable because they were unaware of different kinds of available online mental health resources and therefore did not think online resources could be helpful.

It is notable that more than half of control participants did not feel comfortable finding online mental health resources independently. However, they suggested and accessed similar online depression resources the intervention group received. A majority of participants in both groups found anonymity, supportiveness, and accessibility to be helpful characteristics in an online mental health resource. These findings are similar to previous online intervention studies for young people $[9,22]$. Of importance, the intervention resource list encompassed the characteristics mentioned above.

\section{Limitations}

There were several limitations to the study. Only one social media site was used and the sample size was small, therefore findings cannot be generalized; however, this study was intended to test a research protocol and determine whether it could be used in future studies. Due to profile deletion and nonresponse to the survey, the intervention group had only female participants. However, males and females are equally likely to post a reference to depression on social media [12,29]. A previous depression intervention study did not find a statistically significant difference in the success of the intervention between males and females [3]. The deletion of several profiles in the course of this study was unexpected, further study could investigate whether other social media sites promote more stable or long-lasting profiles. Due to the anonymity of the surveys and the priority to protect participant identities, researchers had no capacity to follow up if participants answered that they had thoughts self-harm or suicide. The anonymity of the survey also prevented researchers from contacting participants to remind them to take the survey. This could be an explanation for the low percentage of completed surveys.

\section{Future Studies}

Findings support future work to test this approach and should consider the following:

- Obtain larger sample sizes with less restrictive inclusion criteria. Many profiles were not able to be included due to the age, language, and privacy restrictions.

- Utilize other social media sites such as Facebook or Twitter to reach a larger population of social media users. 
- Follow up with participants for feedback on why they found the intervention and certain resources to be helpful or not helpful and whether this approach was considered intrusive. This would allow a mental health expert to verify a resource list more suitable for young people.

\section{Conclusions}

Previous successful online intervention studies have not incorporated social media for the identification and recruitment of participants or to offer mental health resources. Findings support the need for mental health resources targeting the adolescent and young adult population, and this study offers a novel approach for offering these services. Social media is an appropriate platform for mental health interventions for young people because it can reach a diverse population while remaining cost effective. This intervention protocol could be utilized and expanded in future studies to further understand resources utilized by young people and find new approaches to reaching this population.

\section{Acknowledgments}

This research was supported and funded by the Seattle Children's Research Institute.

\section{Conflicts of Interest}

None declared.

\section{Editorial Notice}

This randomized study was not registered, explained by authors as the study not meeting criteria as Applicable Clinical Trial (ACT) Under 42 CFR 11.22(b). The editor granted an exception from ICMJE rules mandating prospective registration of randomized trials as he considered the study formative. However, readers are advised to carefully assess the validity of any potential explicit or implicit claims related to primary outcomes or effectiveness, as lack of registration does not prevent authors from changing their outcome measures retrospectively.

\section{Multimedia Appendix 1}

Resources sent to intervention participants.

[JPG File, 288KB-Multimedia Appendix 1]

\section{Multimedia Appendix 2 \\ CONSORT-EHEALTH checklist (V 1.6.1).}

[PDF File (Adobe PDF File), 568KB-Multimedia Appendix 2]

\section{References}

1. Kazdin AE. Addressing the treatment gap: A key challenge for extending evidence-based psychosocial interventions. Behav Res Ther 2017 Jan;88:7-18. [doi: 10.1016/j.brat.2016.06.004] [Medline: 28110678]

2. Mojtabai R, Olfson M, Han B. National Trends in the Prevalence and Treatment of Depression in Adolescents and Young Adults. Pediatrics 2016 Dec;138(6) [FREE Full text] [doi: 10.1542/peds.2016-1878] [Medline: 27940701]

3. Moreno MA, Christakis DA, Egan KG, Jelenchick LA, Cox E, Young H, et al. A pilot evaluation of associations between displayed depression references on Facebook and self-reported depression using a clinical scale. J Behav Health Serv Res 2012 Jul;39(3):295-304 [FREE Full text] [doi: 10.1007/s11414-011-9258-7] [Medline: 21863354]

4. Patel V, Flisher AJ, Hetrick S, McGorry P. Mental health of young people: a global public-health challenge. Lancet 2007 Apr 14;369(9569):1302-1313. [doi: 10.1016/S0140-6736(07)60368-7] [Medline: 17434406]

5. Eisenberg D, Downs MF, Golberstein E, Zivin K. Stigma and help seeking for mental health among college students. Med Care Res Rev 2009 Oct;66(5):522-541. [doi: 10.1177/1077558709335173] [Medline: 19454625]

6. Hunt J, Eisenberg D. Mental health problems and help-seeking behavior among college students. J Adolesc Health 2010 Jan;46(1):3-10. [doi: 10.1016/j.jadohealth.2009.08.008] [Medline: 20123251]

7. Lenhart A, Page D. Teens, Social Media \& Technology Overview 2015. Washington, DC: Pew Research; 2015. URL: http://assets.pewresearch.org/wp-content/uploads/sites/14/2015/04/PI TeensandTech Update2015 0409151.pdf[WebCite Cache ID 6yL42oB9M]

8. Rickwood DJ, Mazzer KR, Telford NR. Social influences on seeking help from mental health services, in-person and online, during adolescence and young adulthood. BMC Psychiatry 2015;15:40 [FREE Full text] [doi: 10.1186/s12888-015-0429-6] [Medline: 25886609]

9. Naslund JA, Aschbrenner KA, McHugo GJ, Unützer J, Marsch LA, Bartels SJ. Exploring opportunities to support mental health care using social media: A survey of social media users with mental illness. Early Interv Psychiatry 2017 Oct 20. [doi: 10.1111/eip.12496] [Medline: 29052947] 
10. Perrin A. Social Media Usage. Washington, DC: PEW Research; 2015. URL: http://assets.pewresearch.org/wp-content/ uploads/sites/14/2015/10/PI 2015-10-08 Social-Networking-Usage-2005-2015 FINAL.pdf[WebCite Cache ID 6yL4AmW7q]

11. Lachmar EM, Wittenborn AK, Bogen KW, McCauley HL. \#MyDepressionLooksLike: Examining Public Discourse About Depression on Twitter. JMIR Ment Health 2017 Oct 18;4(4):e43 [FREE Full text] [doi: 10.2196/mental.8141] [Medline: $\underline{29046270]}$

12. Moreno MA, Jelenchick LA, Egan KG, Cox E, Young H, Gannon KE, et al. Feeling bad on Facebook: depression disclosures by college students on a social networking site. Depress Anxiety 2011 Jun;28(6):447-455 [FREE Full text] [doi: 10.1002/da.20805] [Medline: 21400639]

13. Moreno MA, Christakis DA, Egan KG, Jelenchick LA, Cox E, Young H, et al. A pilot evaluation of associations between displayed depression references on Facebook and self-reported depression using a clinical scale. J Behav Health Serv Res 2012 Jul;39(3):295-304 [FREE Full text] [doi: 10.1007/s11414-011-9258-7] [Medline: 21863354]

14. Rice S, Robinson J, Bendall S, Hetrick S, Cox G, Bailey E, et al. Online and Social Media Suicide Prevention Interventions for Young People: A Focus on Implementation and Moderation. J Can Acad Child Adolesc Psychiatry 2016;25(2):80-86 [FREE Full text] [Medline: 27274743]

15. Rice SM, Goodall J, Hetrick SE, Parker AG, Gilbertson T, Amminger GP, et al. Online and social networking interventions for the treatment of depression in young people: a systematic review. J Med Internet Res 2014;16(9):e206 [FREE Full text] [doi: 10.2196/jmir.3304] [Medline: 25226790]

16. Tsai Y, Zhou F, Wortley P, Shefer A, Stokley S. Trends and characteristics of preventive care visits among commercially insured adolescents, 2003-2010. J Pediatr 2014 Mar;164(3):625-630. [doi: 10.1016/j.jpeds.2013.10.042] [Medline: 24286572]

17. Whitaker C, Stevelink S, Fear N. The Use of Facebook in Recruiting Participants for Health Research Purposes: A Systematic Review. J Med Internet Res 2017 Aug 28;19(8):e290 [FREE Full text] [doi: 10.2196/jmir.7071] [Medline: 28851679]

18. Deady M, Mills KL, Teesson M, Kay-Lambkin F. An Online Intervention for Co-Occurring Depression and Problematic Alcohol Use in Young People: Primary Outcomes From a Randomized Controlled Trial. J Med Internet Res 2016 Mar 23;18(3):e71 [FREE Full text] [doi: 10.2196/jmir.5178] [Medline: 27009465]

19. Robinson J, Hetrick S, Cox G, Bendall S, Yuen HP, Yung A, et al. Can an Internet-based intervention reduce suicidal ideation, depression and hopelessness among secondary school students: results from a pilot study. Early Interv Psychiatry 2016 Feb;10(1):28-35. [doi: 10.1111/eip.12137] [Medline: 24684946]

20. Robinson J, Hetrick S, Cox G, Bendall S, Yung A, Yuen HP, et al. The development of a randomised controlled trial testing the effects of an online intervention among school students at risk of suicide. BMC Psychiatry 2014 May 27;14:155 [FREE Full text] [doi: 10.1186/1471-244X-14-155] [Medline: 24884888]

21. Naslund JA, Aschbrenner KA, Marsch LA, McHugo GJ, Bartels SJ. Crowdsourcing for conducting randomized trials of internet delivered interventions in people with serious mental illness: A systematic review. Contemp Clin Trials 2015 Sep;44:77-88 [FREE Full text] [doi: 10.1016/j.cct.2015.07.012] [Medline: 26188164]

22. Bold KW, Hanrahan TH, O'Malley SS, Fucito LM. Exploring the Utility of Web-Based Social Media Advertising to Recruit Adult Heavy-Drinking Smokers for Treatment. J Med Internet Res 2016;18(5):e107 [FREE Full text] [doi: 10.2196/jmir.5360] [Medline: 27194456]

23. Moreno MA, Vanderstoep A, Parks MR, Zimmerman FJ, Kurth A, Christakis DA. Reducing at-risk adolescents' display of risk behavior on a social networking web site: a randomized controlled pilot intervention trial. Arch Pediatr Adolesc Med 2009 Jan;163(1):35-41. [doi: 10.1001/archpediatrics.2008.502] [Medline: 19124701]

24. Tumblr. URL: https://www.tumblr.com/[WebCite Cache ID 6yL4XY6Fn]

25. Tumblr. Blog Management URL: https://tumblr.zendesk.com/hc/en-us/sections/205853348-Blog-management[WebCite Cache ID 6yL4mbcph]

26. Bell C. DSM-IV: Diagnostic and Statistical Manual of Mental Disorders. JAMA 1994 Sep 14;272(10):828. [doi: 10.1001/jama.1994.03520100096046]

27. Random.org. URL: https://www.random.org/[WebCite Cache ID 6yL4fhVk1]

28. Richardson LP, McCauley E, Grossman DC, McCarty CA, Richards J, Russo JE, et al. Evaluation of the Patient Health Questionnaire-9 Item for detecting major depression among adolescents. Pediatrics 2010 Dec;126(6):1117-1123 [FREE Full text] [doi: 10.1542/peds.2010-0852] [Medline: 21041282]

29. Egan KG, Koff RN, Moreno MA. College students' responses to mental health status updates on Facebook. Issues Ment Health Nurs 2013 Jan;34(1):46-51. [doi: 10.3109/01612840.2012.718044] [Medline: 23301569]

30. Eysenbach G, CONSORT-EHEALTH Group. CONSORT-EHEALTH: improving and standardizing evaluation reports of Web-based and mobile health interventions. J Med Internet Res 2011 Dec 31;13(4):e126 [FREE Full text] [doi: 10.2196/jmir.1923] [Medline: 22209829]

\section{Abbreviations}

DSM-IV: Diagnostic and Statistical Manual

PHQ-9: Patient Health Questionnaire-9 
Edited by G Eysenbach; submitted 14.11.17; peer-reviewed by S Cash, S Hetrick, JP Allem; comments to author 13.12.17; revised version received 27.02.18; accepted 28.02.18; published 12.04.18

Please cite as:

Kelleher E, Moreno M, Wilt MP

Recruitment of Participants and Delivery of Online Mental Health Resources for Depressed Individuals Using Tumblr: Pilot Randomized Control Trial

JMIR Res Protoc 2018;7(4):e95

URL: http://www.researchprotocols.org/2018/4/e95/

doi: 10.2196/resprot.9421

PMID: 29650507

CErin Kelleher, Megan Moreno, Megan Pumper Wilt. Originally published in JMIR Research Protocols (http://www.researchprotocols.org), 12.04.2018. This is an open-access article distributed under the terms of the Creative Commons Attribution License (https://creativecommons.org/licenses/by/4.0/), which permits unrestricted use, distribution, and reproduction in any medium, provided the original work, first published in JMIR Research Protocols, is properly cited. The complete bibliographic information, a link to the original publication on http://www.researchprotocols.org, as well as this copyright and license information must be included. 